\title{
COVERABLE SEMIGROUPS
}

\author{
A. Y. W. LAU
}

\begin{abstract}
The concept of a semilattice having small semilattices has been studied and some equivalences of this property have been investigated. In the process of investigating semilattices, the author found a class of semigroups called coverable semigroups, and the interesting fact about it is that a necessary and sufficient condition for a compact semilattice to have small semilattices is to be coverable. Also by virtue of the concept of coverable semigroups, one can show that the usual interval is costable instead of the more intricate method of proving it by producing an arc.
\end{abstract}

1. Let $S$ be a topological semigroup and $U_{0}$ be an open cover of $S$. We write $V_{0}<U_{0}$ if $V_{0}$ is a cover of $S$ such that $V_{0}$ refines $U_{0}$ and if $V, U \in V_{0}$, then there exists $W \in V_{0}$ such that $V U \subseteq W$. We shall say that $S$ is (finitely) coverable if given an open cover $U_{0}$ of $S$, there exists a (finite) open cover $V_{0}$ such that $V_{0}<U_{0}$. Furthermore, $S$ is said to be (finitely) neighborable if given an open cover of $S$, there exists a (finite) collection $V_{0}$ of subsets of $S$ such that $V_{0}<U_{0}$ and $S=\bigcup_{V \in V_{0}} V^{0}$ where $V^{0}$ is the interior of $V$ (i.e. union of all open sets contained in $V$ ). A compact semigroup $S$ is costable if given any continuous homomorphism from a compact semigroup $K$ onto $S$, then cd $K \geqq$ cd $S$ where cd is the codimension with respect to some nondegenerate abelian group.

It is obvious that every finitely coverable semigroup is compact and neighborable. Any Hausdorff space given the left trivial multiplication is coverable. The following proposition is in relation to neighborable semigroups.

Lemma 1.1. If $S$ is a neighborable semigroup, then $S$ has small semigroups at $p=p^{2}$ (i.e. if $p$ is in $S$ and $V$ is an open set containing $p$, then there exists a neighborhood $N$ of $p$ such that $N^{2} \subseteq N \subseteq V$ ).

Proof. Let $p \in S$ and $U$ be an open set containing $p$. Then $U_{0}=$ $\{U, S \backslash\{p\}\}$ is an open cover of $S$; hence there exists a cover $V_{0}$ of $S$ such that $V_{0}<U_{0}$ and $S=\bigcup_{V \in V_{0}} V^{0}$.

Received by the editors May 1, 1972.

AMS (MOS) subject classifications (1970). Primary 22A15.

Key words and phrases. Small semilattices, codimension, costable semigroups, totally disconnected semigroups, min intervals, usual intervals.

(c) American Mathematical Society 1973 
Let $p \in V^{0}$ where $V \in V_{0}$. Then $V V \subseteq W_{1}$ for some $W_{1} \in V_{0}$ and $V^{3} \subseteq$ $V W_{1} \subseteq W_{2}$ for some $W_{2} \in V_{0}$. By induction, one can show that if $n$ is a positive integer, then $V^{n} \subseteq W$ for some $W \in V_{0}$. Since $V_{0}<U_{0}$, then $W \subseteq U$ or $W \subseteq S \mid\{p\}$, but $p \in V^{n} \subseteq W$ implies $W \subseteq U$. Hence $V^{n} \subseteq W \subseteq U$. But $n$ is arbitrary; one can conclude $p \in V \subseteq \bigcup_{n \geqq 1} V^{n} \subseteq U$ and $\bigcup_{n \geqq 1} V^{n}$ is a subsemigroup.

In fact, for a compact semilattice the converse of 1.1 is true. Before we prove it, we need some other propositions. The following two lemmas are stated without proof.

LemMa 1.2. If $S$ is a (finitely) coverable (or neighborable) semigroup and $A$ is a closed subsemigroup, then $A$ is (finitely) coverable (or neighborable).

Lemma 1.3 (a topological lemma). If $U_{0}$ is an open cover of $\mathbf{P}_{i \in I} S_{i}$, where each $S_{i}$ is a compact space, then there exists a finite subset $J$ of I and for each $i$ in $J$, there exists a finite open cover $\mathscr{V}_{i}$ of $S_{i}$ such that $\left\{\mathbf{P}_{i \in J} V_{i} \times\right.$ $\left.\mathbf{P}_{i \in I \backslash J} S_{i} \mid V_{i} \in \mathscr{V}_{i}\right\}$ refines $U_{0}$.

Lemma 1.4. If $\left\{S_{i}\right\}_{i \in I}$ is a collection of compact semigroups, then $\mathbf{P}_{i \in I} S_{i}$ is ( finitely) coverable if and only if each $S_{i}$ is (finitely) coverable.

Proof. Suppose $\mathbf{P}_{i \in I} S_{i}$ is (finitely) coverable and $j \in I$. Let $W_{0}$ be an open cover of $S_{j}$. Then $U_{0}=\left\{W \times \mathbf{P}_{i \neq j} S_{i} \mid W \in W_{0}\right\}$ is an open cover of $\mathbf{P}_{i \in I} S_{i}$. There exists $V_{0}$ a (finite) open cover of $\mathbf{P}_{i \in I} S_{i}$ such that $V_{0}<U_{0}$. Then $\left\{\Pi_{j}(V) \mid V \in V_{0}\right\}$ is a (finite) open cover of $S_{j}$, where $\Pi_{j}$ is the $j$ th projection map. It is easy to check $\left\{\Pi_{j}(V) \mid V \in V_{0}\right\}<W_{0}$.

Suppose each $S_{i}$ is (finitely) coverable and $U_{0}$ is an open cover of $\mathbf{P}_{i \in I} S_{i}$. By Lemma 1.3, there exists a finite subset $J$ of $I$ and open cover $\mathscr{V}_{i}$ where $i \in J$ such that $\left\{\mathbf{P}_{i \in J} V_{i} \times \mathbf{P}_{i \in I \backslash J} S_{i} \mid V_{i} \in \mathscr{V}_{i}\right\}$ refines $U_{0}$. But for each $i \in J$, there exists a (finite) open cover $\mathcal{O}_{i}$ such that $\mathcal{O}_{i}<\mathscr{V}_{i}$. Hence

$$
\left\{\underset{i \in J}{\mathbf{P}} O_{i} \times \underset{i \in I \backslash J}{\mathbf{P}} S_{i} \mid O_{i} \in \mathcal{O}_{i}\right\}<\left\{\underset{i \in J}{\mathbf{P}} V_{i} \times \underset{i \in I \backslash J}{\mathbf{P}} S_{i} \mid V_{i} \in \mathscr{V}_{i}\right\}
$$

would yield the result that $\left\{\mathbf{P}_{i \in J} O_{i} \times \mathbf{P}_{i \in I \backslash J} S_{i} \mid O_{i} \in \mathcal{O}_{i}\right\}<U_{0}$. Hence the product is (finitely) coverable.

Lemma 1.5. The min interval (i.e. $[0,1]$ under the multiplication $x y=$ $\min \{x, y\})$ is finitely coverable.

Proof. Let $M$ be the min interval and $U_{0}$ be an open cover of $M$. There exists a Lebesgue number $\delta$ for $U_{0}$. Then $M$ can be covered by a finite number of open intervals or half-open intervals, each of which has length $\delta$. Furthermore, the product of any two such intervals is contained in the interval with the smaller left-hand endpoint. Hence $M$ is finitely coverable. 
THEOREM 1.6. If $S$ is a compact semilattice, then $S$ is coverable if and only if it has small semilattices.

Proof. If $S$ is coverable, by Lemma $1.1, S$ has small semilattices. Suppose $S$ has small semilattices; then [6] showed that $S$ is embeddable topologically and algebraically into a product of min intervals. Since min intervals are coverable, by Lemma 1.4 their product is coverable. By the compactness of $S, S$ can be considered as a closed subsemilattice of the product of min intervals. Hence $S$ is coverable by Lemma 1.2.

2. In this section, we will depart from semilattices and investigate coverable/neighborable semigroups in general. An interesting corollary is that the usual interval is costable.

LeMma 2.1. If $S$ is a neighborable semigroup, then $H(e)$ is totally disconnected for each $e=e^{2}$.

Proof. Let $e=e^{2} \in S$. Suppose $H(e)$ is not totally disconnected. Then there exists a compact connected nondegenerate subgroup $G$ of $H(e)$. Since $G$ is also neighborable, then $G$ has small semigroups at $e$. Let $N$ be a neighborhood of $e$ in $G$ such that $N$ is a proper subsemigroup of $G$. Let $U$ be a set open in $G$ such that $U=U^{-1}$ and $e \in U \subseteq N$. Then $\bigcup_{n \geqq 1} U^{n} \subseteq N$. But this is a contradiction.

LEMMA 2.2. Every compact totally disconnected semigroup is coverable.

Proof. Since every such semigroup is an inverse limit of finite semigroups [4] and finite semigroups are coverable, then an application of Lemmas 1.2 and 1.4 yields the conclusion.

The following theorem shows how neighborable semigroups behave under homomorphisms.

THEOREM 2.3. If $f: S \rightarrow T$ is a continuous closed homomorphism from a ( finitely) neighborable semigroup onto a fully normal semigroup $T$, then $T$ is ( finitely) neighborable.

Proof. Let $C_{0}$ be an open cover of $T$. Then there exists an open cover $U_{0}$ of $T$ such that $U_{0}$ is a star-refinement of $C_{0}$. Since $\left\{f^{-1}(U) \mid U \in U_{0}\right\}$ is an open cover of $S$, there exists a (finite) cover $V_{0}$ of $S$ such that $V_{0}<$ $\left\{f^{-1}(U) \mid U \in U_{0}\right\}$, and $S=\bigcup_{V \in V_{0}} V^{0}$.

For each $y \in T$, define $W_{y}=\left\{V \in V_{0} \mid V \cap f^{-1}(y) \neq \varnothing\right\}$. Then $\left\{f\left(W_{y}\right) \mid y \in T\right\}$ is a (finite) cover of $T$ since $V_{0}$ is a (finite) cover of $S$, and it is obvious that $y \in f\left(W_{y}\right)^{0}$ since $f$ is closed. Now one has to show $\left\{f\left(W_{y}\right) \mid y \in T\right\}<C_{0}$.

Let $y \in T$. Fix $V_{1} \in V_{0}$ such that $V_{1} \cap f^{-1}(y) \neq \varnothing$. Then there exists $U_{1} \in U_{0}$ such that $V_{1} \subseteq f^{-1}\left(U_{1}\right)$, and $U_{1}^{*} \subseteq C$ for some $C \in C_{0}$ since $U_{0}$ is a star-refinement of $C_{0}$. Let $z \in f\left(W_{y}\right)$. Then there exists $V_{2} \in V_{0}$ such that 
$V_{2} \cap f^{-1}(y) \neq \varnothing$ and $z \in f\left(V_{2}\right)$. But $V_{2} \subseteq f^{-1}\left(U_{2}\right)$ for some $U_{2} \in U_{0}$. Hence $y \in f\left(V_{1}\right) \cap f\left(V_{2}\right) \subseteq U_{1} \cap U_{2}$. Since $U_{1} \cap U_{2} \neq \varnothing$, then $U_{2} \subseteq U_{1}^{*} \subseteq C$. Thus $z \in C$ implies $f\left(W_{y}\right) \subseteq C$. Furthermore $f\left(W_{y}\right) f\left(W_{z}\right) \subseteq f\left(W_{y z}\right)$. The proof is complete.

COROLLARY 2.4. If $f: S \rightarrow T$ is a continuous closed homomorphism from a (finitely) neighborable fully normal semigroup $S$ onto a semigroup $T$, then $T$ is (finitely) neighborable.

With a little computation, one can determine that the usual interval $[0,1]$ under real number multiplication is not neighborable. Hence we have the following corollary.

Corollary 2.5. If $f: S \rightarrow I$ is a continuous homomorphism from a compact semigroup $S$ onto the usual interval $I$, then $S$ is not totally disconnected (i.e. I is costable.)

Proof. If $S$ is totally disconnected, then $S$ is coverable. By the previous lemma, $I$ has to be neighborable but it is not. Hence $S$ is not totally disconnected, i.e. codimension of $S \geqq 1$.

The interesting fact about coverable semigroups is that they can have a lot of idempotents (e.g. some semilattices) or they can have very few idempotents (e.g. $\left[0, \frac{1}{2}\right]$ under the usual real number multiplication). The author conjectures that a compact semigroup with zero as its only idempotent is coverable.

\section{REFERENCES}

1. D. R. Brown, Topological semilattices on the two-cell, Pacific J. Math. 15 (1965), 35-46. MR 31 \#725.

2. J. Dugundji, Topology, Allyn and Bacon, Boston, Mass., 1966. MR 33 \#1824.

3. K. H. Hofmann and P. S. Mostert, Elements of compact semigroups, Merrill, Columbus, Ohio, 1966. MR 35 \#285.

4. K. Numakura, Theorems on compact totally disconnected semigroups and lattices, Proc. Amer. Math. Soc. 8 (1957), 623-626. MR 19, 290.

5. A. Y. W. Lau, Small semilattices, Semigroup Forum 4 (1972), 150-155.

6. J. D. Lawson, Topological semilattices with small semilattices, J. London Math. Soc. (2) 1 (1969), 719-724. MR 40 \#6516.

7. J. W. Stepp, Semilattices which are embeddable in a product of min intervals, Semigroup Forum 2 (1971), 80-82.

Department of Mathematics, North Texas State University, Denton, Texas 76203 